\title{
Quantification of Subsurface Damage in a Brittle Insulating Ceramic by Three-Dimensional Focused Ion Beam Tomography
}

\author{
N. Payraudeau, ${ }^{1, \star}$ D. McGrouther, ${ }^{2}$ and K.U. O'Kelly ${ }^{1}$ \\ ${ }^{1}$ Department of Mechanical and Manufacturing Engineering, Trinity College Dublin, Dublin 2, Ireland \\ ${ }^{2}$ S.U.P.A, School of Physics and Astronomy, University of Glasgow, Glasgow G12 8QQ, United Kingdom
}

\begin{abstract}
In this study, we present a fully automated method to investigate and reconstruct the threedimensional crack structure beneath an indent in a highly insulating material. This work concentrates on issues arising from a long automatic acquisition process, the insulating nature of the specimen, and the introduction of minimal damage to the original cracks resulting from indentation.
\end{abstract}

Key words: focused ion beam (FIB) microscopy, crack, microstructure, ceramic, tomography

\section{INTRODUCTION}

In the last decade there have been a significant number of reports illustrating the effectiveness of focused ion beam (FIB) techniques for the characterization of three-dimensional (3D) material structures at length scales ranging from the microto the nanoscale (Inkson et al., 2001; Holzer et al., 2004; Kammer et al., 2005; Uchic et al., 2006; Kato et al., 2007; Schaffer et al., 2007; Matthijs De Winter et al., 2009). An area of materials science in which these techniques have proved particularly useful has been the investigation of subsurface damage following the intentional contact of the material surface by a loaded indenter (Steer et al., 2001, 2002; Inkson et al., 2006; Xie et al., 2006; Elfallagh \& Inkson, 2008). Using the FIB, ultrasmooth subsurface cross sections can be prepared through the contact site allowing the extent and nature of the underlying damage to be assessed. Most work of this type has been performed on metals and semiconductors due to the practical ease with which they can be sectioned in the FIB (Claves et al., 2004; Williams et al., 2004; Kammer et al., 2005; Uchic et al., 2006; Williams et al., 2006; Holzapfel et al., 2007; Kato et al., 2007; McGrouther \& Munroe, 2007). With rather more difficulty, these methods have been applied to insulators including biological materials or ceramics (Holzer et al., 2004; Schaffer et al., 2007; Matthijs De Winter et al., 2009).

Inkson et al. (2001) introduced the method commonly referred to as FIB tomography. The technique involves using the ion beam to first open a trench exposing the material in cross section. A serial sectioning approach is then performed where the ion beam is used to remove a controlled amount of material followed by the capture of an image of the resulting cross-section face. The imaging step can be performed using the ion column [producing ion-induced secondary electrons (I-SEs)], but on the dual-column instruments that are becoming widespread now, it is more nor-

Received September 22, 2010; accepted December 15, 2010

${ }^{\star}$ Corresponding author. E-mail: payraudn@tcd.ie mally acquired using the scanning electron microscope (SEM) column [generating electron-induced secondary electrons (E-SEs)]. The process of material removal and imaging is repeated until a sequence of images is acquired over the whole volume of the region of interest (ROI).

When FIB tomography is applied to poorly conducting or completely insulating materials, major difficulties can arise due to charging effects during both the material removal and imaging steps. Several authors have described these and proposed refinements to Inkson's original technique (Holzer et al., 2004; Schaffer et al., 2007; Matthijs De Winter et al., 2009). In this article we describe the particular set of difficulties faced when utilizing FIB tomography to investigate the influence of microstructure on cracking under nanoindents in highly insulating SiAlON ceramics. By investigating the phenomena related to electrical charging, we have developed a fully automated method capable of producing reliable image datasets for 3D reconstruction, and this has provided new insight into the fracture mechanics of brittle ceramic materials.

\section{Materials and Methods}

The material sample investigated was a Syalon 101 ceramic (Internationals Syalons, Wallsend, England). Microstructurally, the ceramic was composed of grains of $\beta$-SiAlON $\left(\mathrm{Si}_{5.5} \mathrm{Al}_{0.5} \mathrm{O}_{0.5} \mathrm{~N}_{7.5}\right)$ with sizes distributed in the range 1 to $3 \mu \mathrm{m}$ and separated by an intergranular yttrium-aluminumoxynitride glass. The sample also included numerous voids with sizes also in the range of microns.

In brittle materials, fatigue and failure are based on the initiation and propagation of microcracks, two phenomena that are strongly influenced by the intrinsic microstructure. In this work, indentation was used to induce plastic deformation and cracking as a relevant tool to the study of contact-induced damage occurring during machining and wear. A MTS NanoIndenter XP (Agilent Technologies, Santa Clara, CA, USA) was used to indent the ceramic applying a 


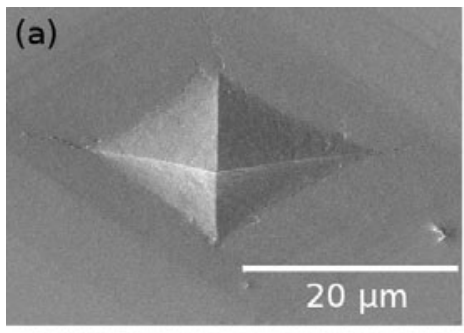

(c)
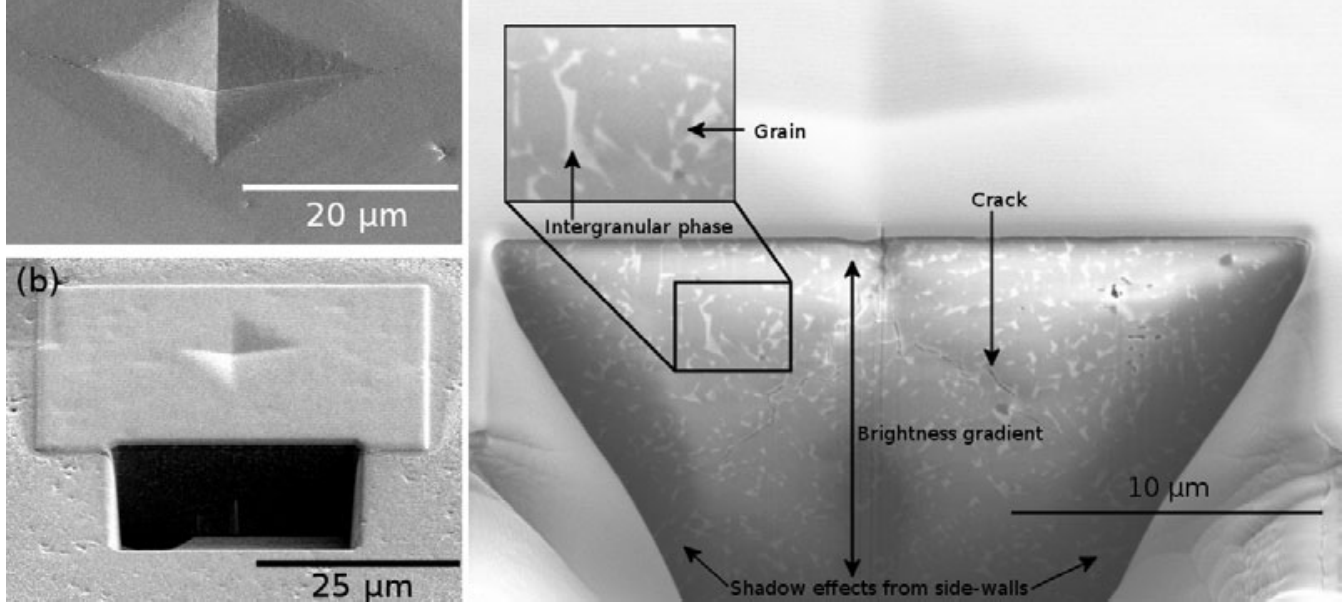

Figure 1. Micrograph of a $2 \mathrm{~N}$ indent sectioned using the FIB. The relevant contrast features in the image are indicated (phases, cracks, charging features, etc.).

Vickers diamond indenter tip at loads ranging from $0.5 \mathrm{~N}$ to $5 \mathrm{~N}$. Figure 1a shows a tilted E-SE image of an indent resulting from an applied load of $2 \mathrm{~N}$.

Prior to serial sectioning of the material volume under the indent, a $30 \mathrm{~nm}$ thick $\mathrm{Au}$ surface film was sputter deposited to minimize electrical charging of the top surface. Serial sectioning and imaging of the sample were performed in an FEI Nova Nanolab 200 Dualbeam instrument equipped with the Sidewinder $\mathrm{Ga}^{+}$ion column operating at $30 \mathrm{keV}$ and a field emission gun SEM. For our task the manufacturer provided Slice \& View software tool was completely inadequate in handling problems associated with sectioning a highly insulating ceramic, as we describe below, so we modified our own feature enhanced serial sectioning script platform.

As a first step, the area of interest was protected from damage by depositing a $1 \mu \mathrm{m}$ platinum layer using the in situ gas injector system deposition. A trench, immediately adjacent to the Pt protection pad and approximately $26 \mu \mathrm{m}$ wide, was opened up using an ion beam current of $21 \mathrm{nA}$ to expose the material subsurface (as depicted in Fig. 1b). Subsequently, slices were milled using an ion beam current of $3.0 \mathrm{nA}$ at $100 \mathrm{~nm}$ intervals and the cross-section face exposed by each imaged using the SEM. The slice interval (thickness) was considered to be sufficient for effective reconstruction of the $\beta$-SiAlON grains with sizes in the range 1 to $3 \mu \mathrm{m}$ and the related cracks under the indent.

The serial sectioning and imaging process produced a stack typically consisting of 200 images. A combination of software packages was used to perform the tricky reconstruction process. The relative alignment of the images within the stack was initially adjusted using the slice alignment tool of Avizo (Visualization Sciences Group, Burlington, MA, USA) 3D visualization software. Segmentation of the stack was performed by a twin approach.

Prior to the identification of the $\beta$-SiAlON grains and the intergranular phase using the segmentation editor in
Avizo, the FIB images were processed in Matlab with a customized script to remove brightness gradients due to charging effects. Segmentation of the cracks was more effectively performed by importing the stack into IMOD [IMOD from the University of Colorado-Boulder (Kremer et al., 1996)]. Finally, 3D visualization of the reconstructed microstructure combined with the crack system was performed by recombining both of the segmented stacks in Avizo.

\section{Results And Discussion}

\section{Initial Sectioning and Optimization of SEM Imaging Conditions}

Our first experiment to investigate subsurface cracking involved cutting a trench with the ion beam midway through a Pt protected indent and acquiring E-SE images to view the microstructure. Figure 1c shows the result of sectioning through the indent where both the structural features of interest are indicated along with a number of phenomena related to the highly insulating nature of the material.

Viewing the newly cut cross-section image, Figure 1c, distortions and strong voltage contrast (strong bright and dark regions appearing on the flat cross-section face due to non-even charge distribution) were observed. In an attempt to minimize this effect, the energy of the incident electron beam and the image scan parameters were varied. The former approach was employed to try to balance the primary charge incident upon the cross-section face during scanning with that leaving the face in the form of secondary and backscattered electrons (Goldstein et al., 2003; Holzer et al., 2004). An electron beam energy of $5 \mathrm{keV}$ was found to offer good spatial resolution imaging, sufficient to resolve clearly the microscale cracks and to be the best compromise between reducing levels of voltage contrast and maximizing the contrast needed to differentiate the amorphous and crystalline phases. Further reduction in the levels of voltage contrast due to electrical charging was obtained by choosing a low 


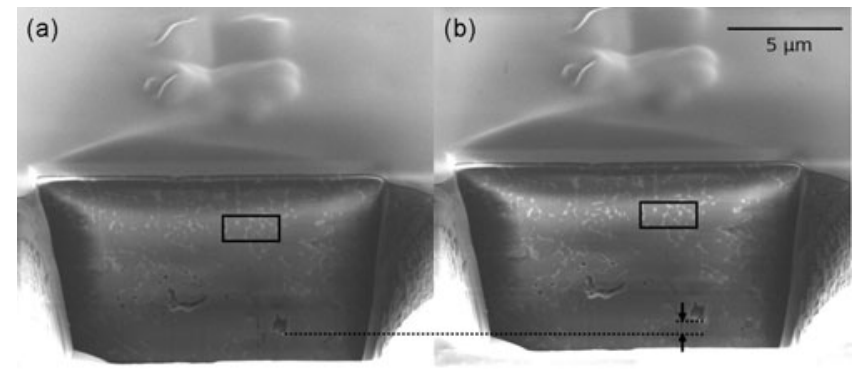

Figure 2. Micrographs of an indent cross-section face (a) after the 1 st image and (b) after the 20th image. $212 \times 94 \mathrm{~mm}(150 \times 150 \mathrm{DPI})$.

beam current $(0.40 \mathrm{nA}$, to reduce the amount of implanted charge) and by using a relatively quick scan rate (dwell time $=$ $3 \mu \mathrm{s})$ averaged over 16 frames. Images acquired, in which less charge was implanted (dwell time $=30 \mu \mathrm{s}$ ), over a single frame exhibited far stronger voltage contrast.

One unwanted contrast feature that the optimization of beam energy, current, and image acquisition parameters could not solve was the presence of shadow effects from the side walls, indicated in Figure 1. This effect, which obscures important microstructural and crack features, is due to charging and is material dependent. A suggested approach to overcome this problem is by milling a "U-shaped" trench around the investigated sample location prior to the serial sectioning process (Holzer et al., 2004; Schaffer et al., 2007; Matthijs De Winter et al., 2009). However, for our study of subsurface damage, this technique is unsuitable as the creation of the U-pattern would induce significant changes in the stress fields surrounding the indent and lead to the opening or closing of cracks through stress relief.

To gauge dynamically the effects of charge build-up using the most optimal imaging conditions $(5 \mathrm{keV}, 0.40 \mathrm{nA}$, 16 frames, $3 \mu$ s dwell time), a newly cut cross section was imaged 20 times. As a result of repeated imaging of the same face, drifts of the image location and changes in the intensity levels in the images were observed between the 1st and the 20th images and can be seen by comparing Figures $2 \mathrm{a}$ and $2 \mathrm{~b}$. They are analyzed and are presented in Figure 3.

Repeated E-SE imaging led to a marked increase in the overall intensity of the pixels within the cross-section face. The mean intensity values were calculated for the region indicated by the dashed box in Figure 2 and plotted in Figure 3. The mean intensity of the region increased approximately linearly up to image 10 with the effect weakening after this as a plateau was reached. Between the 1st and the 20th image, the mean intensity of the region increased by $24 \%$. We assume that such an increase in intensity arises from implantation of a net negative charge with each image acquisition. Each image results in the cross-section face being at an increasingly more negative potential than the surroundings and having the effect that the incident electrons are decelerated as they approach. Incident electrons with lower kinetic energies favor higher secondary electron emission and lead to an increase in image intensity. Further-

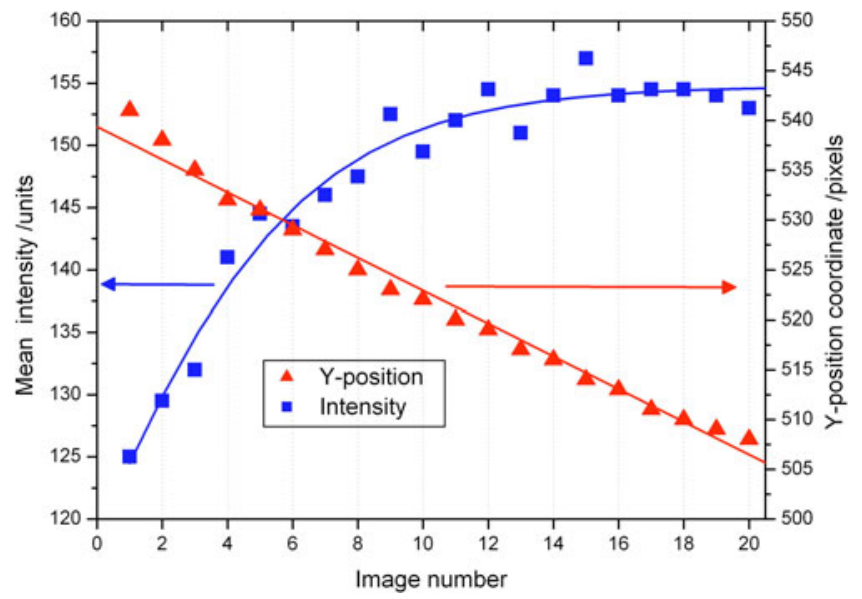

Figure 3. Graph showing changes in mean intensity and position of the selected region against image number. $594 \times 419 \mathrm{~mm}$ $(150 \times 150$ DPI $)$.

more, while this phenomenon was observed after repeated E-SE imaging, it is also likely to be a problem during serial sectioning or ion (I-SE) imaging where the positive charge imparted by the $\mathrm{Ga}^{+}$ion beam milling would not necessarily be expected to cancel the negative charge induced by E-SE imaging.

Figure 3 also showed a linear displacement of the image along the $y$-axis direction. This was again attributed to the accumulation of negative charge on the cross section leading to a deflection of the incident electron beam. This behavior highlighted the potential sensitivity of both the ion and electron beam positioning to the local electrostatic conditions and indicated that a method of drift correction would be needed during the slicing process.

\section{Serial Sectioning of the Microindented Material Volumes}

The single sectioning of the micro-indent provided a great deal of information about the difficulties caused by electrostatic charging of the SiAlON ceramic material. From these observations it was clear that to develop an automated serial sectioning routine that would yield image data of high enough quality for $3 \mathrm{D}$ reconstruction, dynamic adjustment of both the FIB and SEM processes would be required.

Prior to serial sectioning, similar preparations were made (Pt layer deposition, milling of the trench, etc.) as described for the section performed midway through the indent. In this case, however, the initial trench was milled at a position approximately $18 \mu \mathrm{m}$ from the indent center.

\section{Details of the FIB Slicing Process}

A beam current of $3.0 \mathrm{nA}$ was chosen for the milling of the slices. This was based on choosing the lowest beam current possible to mill volume of material $26^{*} 30^{*} 8 \mu \mathrm{m}^{3}$ in a timescale of around $12-15 \mathrm{~h}$ (for a run taking place overnight).

Commonly, when performing serial sectioning with the manufacturer-supplied software, the ion beam automatically 

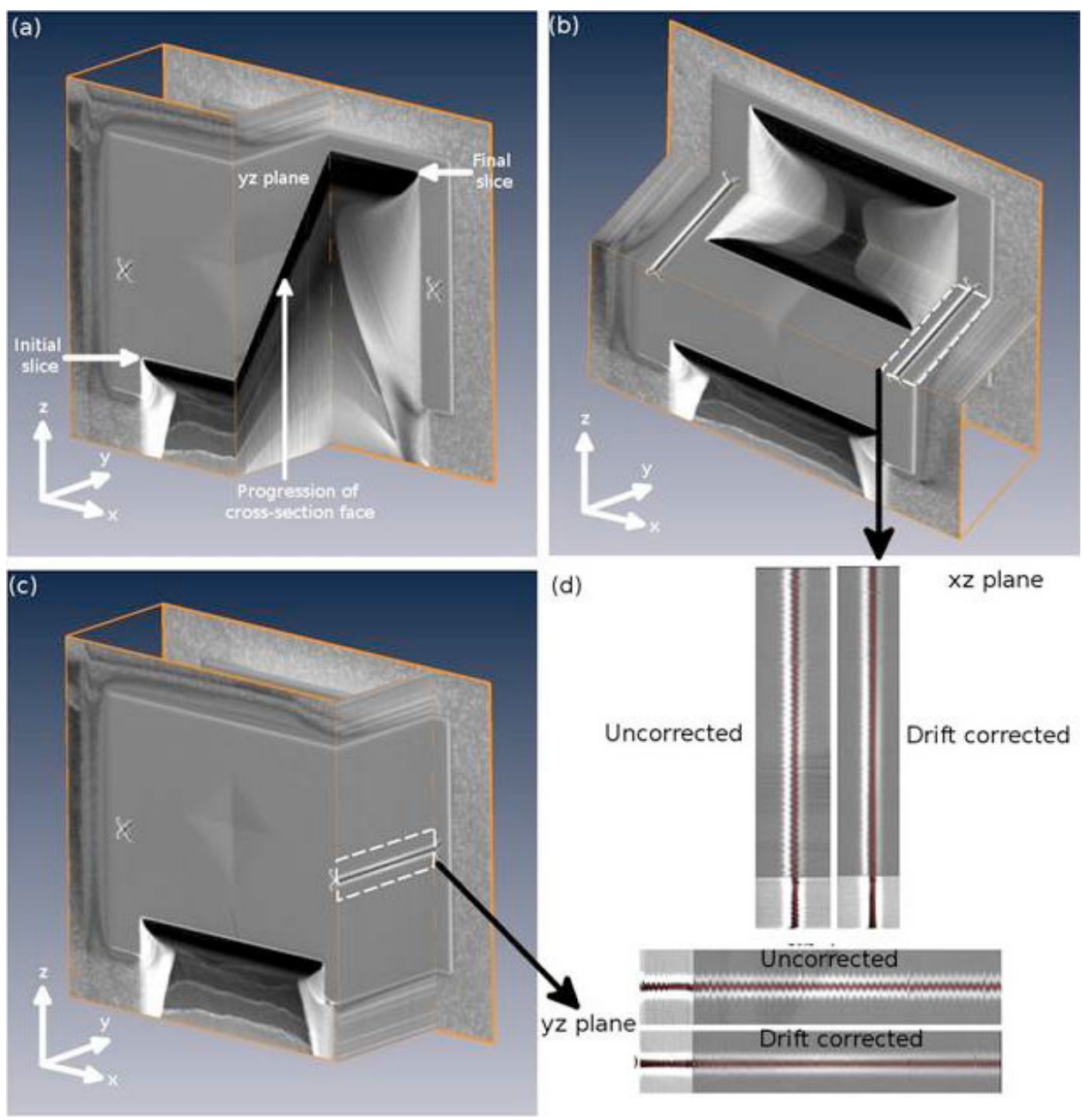

Figure 4. Analysis of ISE images. a: $y z$ cut plane through stack of drift-corrected I-SE images showing the smooth progression of the cross-section face. $\mathbf{b}, \mathbf{c}: x z$ and $y z$ cut planes through right-most reference cross of drift-corrected I-SE image stack. d: Comparison of $x z$ and $y z$ cut planes through the reference cross for uncorrected and drift-corrected I-SE image stacks highlighting the need for drift correction. $111 \times 111 \mathrm{~mm}(150 \times 150 \mathrm{DPI})$.

mills slices of a specified thickness and assumes that the specimen stays effectively in the same position throughout the process. This assumption is a critical one, as any change in the effective specimen position will lead to irregularity of the slice thickness and affect the spatial resolution of the 3D reconstruction. For any material type, a poor mounting of the sample or mechanical drift of the sample stage could cause a variation in this effective position. These effects are usually minimal due to secure sample mounting and the fact that the ratio of the slice thickness/slice milling time is usually larger than the nominal mechanical drift rate. For our highly insulating samples, the initial observation of beam deflection due to local electrostatic charging suggested that some method of ion-beam position checking and realignment would be required to maintain the consistency of slice thickness. For this purpose, a pair of reference markers, crosses, was milled outside of the ROI and images of them recorded. Prior to the milling of each slice, our script acquired an ion beam secondary electron image and utilized image recognition to assess the strength of the beamshift drift correction to be applied. As the volume of the slices to be milled was large, a relatively high ion beam current, $3.0 \mathrm{nA}$, was employed. Repeated imaging of the crosses using such a large beam current resulted in gradual erosion so that a method of periodically updating the reference images was also employed.

The effectiveness of the drift-correction procedure and resulting quality of the slicing can be analyzed by forming image stacks from both the uncorrected and drift-corrected I-SE images. Figure 4 shows clearly the necessity for the automated drift-correction procedure. The main image in Figure 4a shows the I-SE plan-view image (the $x y$ plane) of the material volume at the start of the slicing process. Overlaid are orthogonal lines through one of the reference crosses indicating the positions from which the $x z$ and $y z$ projection image sets in Figure $4 b, c$ are formed. For each of the $x z$ and $y z$ planes, the projection images show presence and effective correction of the ion beam drift over the entire slicing sequence. The stack of I-SE images can be further used to check the variation in thickness of the milled slices 


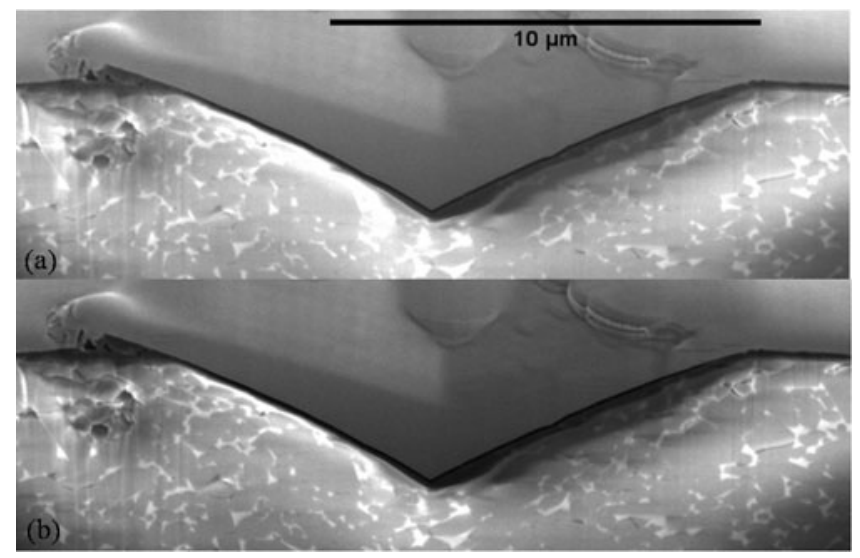

Figure 5. Electron beam images taken (a) without and (b) with brightness correction. $194 \times 124 \mathrm{~mm}(150 \times 150 \mathrm{DPI})$.

(this is critical to the spatial resolution of the reconstruction along the slicing direction). By forming a sum of projection images in the $x z$ plane from the region of the trench headwall, its displacement with each slice can be checked. Doing this confirmed that the slice thickness, $100 \mathrm{~nm}$, was constant throughout the process.

\section{Details of the E-SE Imaging Process}

The conditions used for SEM imaging of the cross-section face after each slice were the optimal conditions discussed previously ( $5 \mathrm{keV}, 0.40 \mathrm{nA}, 3 \mu$ s dwell, 16 frames average). The focus of the electron beam was automatically adjusted to compensate for the slight increase in working distance that occurs as the cross-section face is sequentially milled.

From the observations made during the initial repeated E-SE imaging experiment, it was expected that electrical charging would exert a significant influence upon the imaging during the serial sectioning procedure too. While this was the case, the situation here was improved. The slice milling and imaging procedure inflicts first positively charged ions then negatively charged electrons upon the cross-section face. While the charges are indeed opposite, it would not be expected that they should cancel each other out. During the serial sectioning procedure, it was observed that the level of drift of the E-SE images was small enough that it could be corrected for later in postprocessing of the E-SE image stack. Therefore, drift correction of the E-SE images was not performed in order to reduce the overall run time. Again, as for the initial repeated imaging experiment, a problematic linear increase in brightness was observed to occur. This resulted in saturation of areas of the E-SE images, obscuring both microstructural and crack features, in the latter part of the serial sectioning sequence. To deal with this, two E-SE images were acquired per slice, the first using the detector amplifier conditions (contrast and brightness) set at the start of the run and the second utilizing a compensating brightness reduction. The magnitude of the brightness reduction was empirically determined over several sectioning runs and applied as a linear correction with slice number. Figure 5 shows

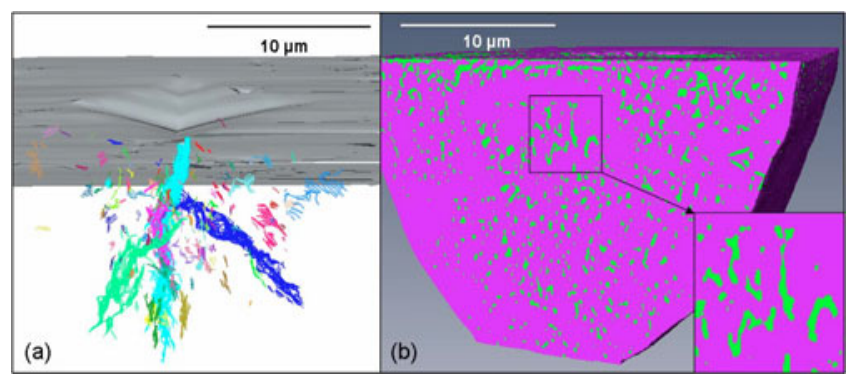

Figure 6. a: Reconstruction of cracks underneath a $2 \mathrm{~N}$ indent in Syalon 101. Each individual line is a contour from each individual slice, and each color represents a different crack. The surface is in gray. b: Reconstruction of the microstructure. $209 \times 91 \mathrm{~mm}$ $(150 \times 150$ DPI $)$.

two electron beam images of the same area of interest: (a) one taken without brightness correction and (b) one with. It can be seen that the automated reduction of brightness has avoided obscuration of image features due to saturation.

\section{3-D Reconstruction}

After tilt correction, E-SE images were aligned using Avizo, and subsurface crack contours were drawn on each slice using the image analysis software IMOD (Fig. 6a) while the microstructure was reconstructed using Avizo (Fig. 6b).

In reconstructing the microstructure, two major difficulties were encountered that arose from charging effects: a brightness gradient from the top to the bottom of the cross section and shadow effects from the side walls. These two phenomena induce difficulties during segmentation by introducing different gray levels within the same phase. Thus, data segmentation by a global threshold value is no longer possible. As only a limited number of filters were available for segmentation in Avizo, a customized script was created in Matlab to overcome this issue. This script used linear filters and two local thresholds to transform the gray level images to binary ones (with the intergranular phase in white and the grains in black). Then a filter to remove small objects (noise) was used, and the binary images obtained were superimposed to the original ones to check the filtering process. Binary images were then segmented in Avizo using a global threshold.

Reconstruction of subsurface cracks was manually performed on each slice. Individual cracks were identified and tagged, and the crack surface area was calculated by interpolating between consecutive slices. As the $z$-projections showed a constant slice thickness of $100 \mathrm{~nm}$, an estimation of the crack surface area was possible and gave a value of $340 \mu \mathrm{m}^{2}$.

The calculations above assume that all cracks observed are resulting from the indentation experiment. However, they do not take into account the fact that FIB sputtering induces changes in residual stress fields. By opening a new free surface, local residual stresses are reduced that leads to an opening or a closing of existing cracks. Moreover, it has 
been shown (Inkson et al., 2006; Elfallagh \& Inkson, 2008) that during an unidirectional serial sectioning, the second half of the indent site presents a lower density of cracks than the first half, due to the residual stress changes during FIB tomographic analysis. This change in local residual stresses can lead to an overestimation of the actual crack surface area when reconstructing crack paths using the image analysis software. The development of a method to estimate the percentage of cracks being opened or closed during the FIB process is currently in progress. This will permit the calculation of the true area of cracks opened during indentation, leading to an estimation of the energy dissipated during the cracking process.

\section{CONCLUSIONS}

Through developing a highly flexible script platform, we have been able to investigate, by FIB serial sectioning, the subsurface damage induced by indentation in a brittle highly insulating ceramic. As we have described, the variety of effects due to electrical charging posed the greatest difficulty for the automated serial sectioning procedure. By overcoming these, reconstruction of the $3 \mathrm{D}$ volume under the indent, including both the microstructure and the cracks, was performed allowing visualization of the crack structure and quantification of its surface area.

\section{ACKNOWLEDGMENTS}

This work was supported by SFI (Science Foundation Ireland). The authors also acknowledge financial support from the European Union under the Framework 6 program under a contract for an Integrated Infrastructure Initiative (Reference 026019 ESTEEM).

\section{REFERENCES}

Claves, S.R., Bandar, A.R., Misiolek, W.Z. \& Michael, J.R. (2004). Three-dimensional (3D) reconstruction of AlFeSi intermetallic particles in 6xxx aluminum alloys using the focused ion beam (FIB). Microsc Microanal 10, 1138-1139.

Elfallagh, F. \& InKson, B.J. (2008). 3D tomographic analysis of crack morphologies in alumina and glass using FIB microscopy. J Phys Conf Ser 126, 1-4.

Goldstein, J., Newbury, D., Joy, D., Lyman, C., Echlin, P., Lifshin, E., Sawyer, L. \& Michael, J. (2003). Scanning Electron Microscopy and X-ray Microanalysis. New York: Springer.

Holzapfel, C., Schaf, W., Marx, M., Vehoff, H. \& Mucklich, F. (2007). Interaction of cracks with precipitates and grain boundaries: Understanding crack growth mechanisms through focused ion beam tomography. Scripta Mater 56, 697-700.
Holzer, L., Indutnyi, F., Gasser, P.H., Munch, B. \& Wegmann, M. (2004). Three-dimensional analysis of porous $\mathrm{BaTiO}_{3}$ ceramics using FIB nanotomography. J Microsc 216, 84-95.

Inkson, B.J., Leclere, D., Elfallagh, F. \& Derby, B. (2006). The effect of focused ion beam machining on residual stress and crack morphologies in alumina. J Phys Conf Ser 26, 219-222.

Inkson, B.J., Wu, H.Z., Steer, T. \& Möвus, G. (2001). 3D mapping of subsurface cracks in alumina using FIB. Mater Res Soc Symp Proc 649, Q3.7.

Kammer, D., Mendoza, R., Barnett, S.A. \& Voorhees, P.W. (2005). The three-dimensional microstructure of materials: Measurement and analysis. Microsc Microanal 11, 72-73.

Kato, M., Ito, T., Aoyama, Y., Sawa, K., Kaneko, T., Kawase, N. \& JinnAI, H. (2007). Three-dimensional structural analysis of a block copolymer by scanning electron microscopy combined with a focused ion beam. J Polymer Sci B: Polymer Phys 45, 677-683.

Kremer, J.R., Mastronarde, D.N. \& McIntosh, J.R. (1996). Computer visualization of three-dimensional image data using IMOD. J Struct Biol 116, 71-76.

Matthijs De Winter, D.A., Schneijdenberg, C.T.W.M., Lebbink, M.N., Lich, B., Verkleij, A.J., Drury, M.R. \& Humbel, B.M. (2009). Tomography of insulating biological and geological materials using focused ion beam (FIB) sectioning and low-kV BSE imaging. J Microsc 233, 372-383.

McGrouther, D. \& Munroe, P.R. (2007). Imaging and analysis of 3-D structure using a dual beam FIB. Microsc Res Techniq 70, 186-194.

Schaffer, M., Wagner, J., Schaffer, B., Schmied, M. \& MulDERs, H. (2007). Automated three-dimensional X-ray analysis using a dual-beam FIB. Ultramicroscopy 107, 587-597.

Steer, T.J., Möbus, G., Kraft, O., Wagner, T. \& Inkson, B.J. (2001). 3D FIB and AFM mapping of nanoindentation zones. Mater Res Soc Symp Proc 649, 3.3.1-3.3.6.

Steer, T.J., Möbus, G., Kraft, O., Wagner, T. \& Inkson, B.J. (2002). 3-D-focused ion beam mapping of nanoindentation zones in a Cu-Ti multilayered coating. Thin Solid Films 413, 147-154.

Uchic, M.D., Groeber, M.A., Dimiduk, D.M. \& Simmons, J.P. (2006). 3D microstructural characterization of nickel superalloys via serial-sectioning using a dual beam FIB-SEM. Scripta Mater 55, 23-28.

Williams, R., Bhattacharyya, D., Viswanathan, G.B., BanerJeE, R. \& Fraser, H.L. (2004). Application of FIB-tomography to the study of microstructures in titanium alloys. Microsc Microanal 10(S2), 1178-1179 (CD-ROM).

Williams, R., Uchic, M., Dimiduk, D. \& Fraser, H.L. (2006). Three dimensional reconstruction of alpha laths in alpha/beta titanium alloys by serial sectioning with a FEI NOVA 600 . Microsc Microanal 12(S2), 1234-1235 (CD-ROM).

Xie, Z.H., Munroe, P.R., McGrouther, D., Singh, R.K., Hoffman, M., Bendavid, A., Martin, P.J. \& Yew, S. (2006). Three-dimensional study of indentation-induced cracks in an amorphous carbon coating on a steel substrate. J Mater Res 21, 2600-2610. 